\title{
T2-Weighted Endorectal Magnetic Resonance Imaging of Prostate Cancer after External Beam Radiation Therapy
}

\author{
Antonio C. Westphalen, John Kurhanewicz, Rui M. G. Cunha, I-Chow Hsu, John Kornak, \\ Shoujun Zhao, Fergus V. Coakley
}

Department of Radiology, Abdominal Imaging Section, University of California San Francisco, San Francisco, California, USA

\begin{abstract}
Purpose: To retrospectively determine the accuracy of T2-weighted endorectal MR imaging in the detection of prostate cancer after external beam radiation therapy and to investigate the relationship between imaging accuracy and time since therapy.

Materials and Methods: Institutional review board approval was obtained and the study was HIPPA compliant. We identified 59 patients who underwent 1.5 Tesla endorectal MR imaging of the prostate between 1999 and 2006 after definitive external beam radiation therapy for biopsy-proven prostate cancer. Two readers recorded the presence or absence of tumor on T2-weighted images. Logistic regression and Fisher's exact tests for $2 \times 2$ tables were used to determine the accuracy of imaging and investigate if accuracy differed between those imaged within 3 years of therapy $(n=25)$ and those imaged more than 3 years after therapy $(n=34)$. Transrectal biopsy was used as the standard of reference for the presence or absence of recurrent cancer.

Results: Thirty-four of 59 patients (58\%) had recurrent prostate cancer detected on biopsy. The overall accuracy of T2weighted MR imaging in the detection cancer after external beam radiation therapy was 63\% (37/59) for reader 1 and $71 \%$ for reader 2 (42/59). For both readers, logistic regression showed no difference in accuracy between those imaged within 3 years of therapy and those imaged more than 3 years after therapy $(p=0.86$ for reader 1 and 0.44 for reader 2$)$.

Conclusion: T2-weighted endorectal MR imaging has low accuracy in the detection of prostate cancer after external beam radiation therapy, irrespective of the time since therapy.
\end{abstract}

Key words: prostate cancer; radiotherapy; follow-up studies; magnetic resonance imaging Int Braz J Urol. 2009; 35: 171-82

\section{INTRODUCTION}

Approximately $30 \%$ of patients with newly diagnosed prostate cancer undergo external beam radiation therapy (EBRT) as their initial definitive treatment (1). Up to $50 \%$ of these patients develop biochemical failure (rising serum prostatic-specific antigen [PSA] after a nadir level has been reached) within 5 years, depending on pre-treatment risk fac- tors $(2,3)$. Biochemical failure may be due to local or systemic recurrence or both (3). Irrespective of the PSA trend, identification of tumor in the treated gland early after completion of radiation therapy is important, because the presence of tumor at needle biopsy performed 2-3 years after radiation, even in patients without clinical or biochemical recurrence, is an important predictor of long-term outcome $(4,5)$. However, a non-invasive alternative to transrectal 
biopsy would clearly be preferable for post-radiation monitoring. Over the last decade, MR imaging has emerged as a powerful tool for locoregional evaluation of prostate cancer. The use of MR imaging after radiation therapy is controversial because post-radiation changes such as prostatic atrophy, the development of diffuse low T2 signal intensity, and indistinctness of the normal zonal anatomy might adversely impact the accuracy of T2-weighted MR imaging (6-8). To our knowledge, only five other studies that in total enrolled just 146 patients have previously investigated the method in this same setting, with inconsistent results that range from low to moderate accuracy (9-13). The existing literature has not systematically reported the influence of time since therapy on the accuracy of MR imaging, although there are good reasons to believe this might be an important variable. For example, it is likely that post-radiation MR changes are at least in part reversible. Pickett et al. showed that 26 months or more after EBRT, $60 \%$ of patients present with areas of the prostate that have normal metabolism on serial MR spectroscopic imaging (14). It is conceivable that the diverging results reported by prior studies are influenced by the time interval since radiation. Therefore, we undertook this study to retrospectively determine the accuracy of T2weighted endorectal MR imaging in the detection of prostate cancer after external beam radiation therapy, and to investigate the relationship between imaging accuracy and time since therapy.

\section{MATERIAL AND METHODS}

\section{Patients}

This was a retrospective single institution study approved by our Committee on Human Research with waiver of informed consent. The study was compliant with requirements of the Health Insurance Portability and Accountability Act. We retrospectively identified, through a cross-correlated computerized search of our medical and radiology information systems, all patients who met the following inclusion criteria:

1 - Definitive treatment of biopsy-proven prostate cancer with external beam radiation therapy with or without associated neoadjuvant/adjuvant androgen deprivation therapy.

2 - Post-treatment 1.5 Tesla endorectal MR imaging of the prostate performed between January 1999 and December 2006.

3 - Post-treatment transrectal ultrasound-guided biopsy of the prostate performed within 180 days of MR imaging.

4 - No additional treatment for prostate cancer.

Fifty-nine patients fulfilled these criteria. The information was redacted for bind review. Eleven of these men were included in a prior preliminary study investigating the use of MR imaging and MR spectroscopic imaging for detection of tumor after radiation therapy (9).

The study group consisted of 59 men with a mean age of 68.8 years (range, 45.2 to 81.6 ), a mean pretreatment serum PSA level of $18.2 \mathrm{ng} / \mathrm{mL}$ (range, 3.5 to 93.0), and the following pretreatment clinical stage (American Joint Committee on Cancer) established on digital rectal examination: $\mathrm{T} 1(\mathrm{n}=9 / 59,15.3 \%), \mathrm{T} 2(\mathrm{n}$ $=31 / 59,52.5 \%), \mathrm{T} 3(\mathrm{n}=14 / 59,23.7 \%)$, or unknown (n $=5 / 59,8.5 \%$ ). The median Gleason score was 7 (range, 5 to 9). The D'Amico risk stratification was based on the clinical stage, PSA level, and Gleason score (15). Patients were categorized as having low risk $(n=7 / 59$, $11.9 \%)$, intermediate risk ( $\mathrm{n}=26 / 59,44.1 \%$ ), or high risk $(n=26 / 59,44.1 \%)$ tumor.

Forty-two patients received a mean dose of 74.6 Gy (range, 65-82 Gy); the dose administered to 17 patients treated at outside institutions was unknown, but all completed a full course of standard radiotherapy. Seventeen patients $(17 / 59,28.9 \%), 5$ $(5 / 59,8.5 \%)$, and $6(6 / 59,10.2 \%)$ patients underwent neoadjuvant, adjuvant, or neoadjuvant plus adjuvant hormonal therapy for a mean duration of 3.9 months (range, 2 to 5), 8.6 months (range, 5 to 12), and 13.3 months (range, 4 to 21 ), respectively.

The mean interval from external beam radiation therapy to MR imaging was 44 months (range, 17-138 months), The mean interval between MR imaging and biopsy was 60 days (range, $0-175$ days) and most procedures were performed within 90 days of imaging $(78 \%, 46 / 59)$.

Patients underwent MR imaging to assess suspected local recurrence on the basis of rising PSA. 
At the time of imaging, twenty-two patients (22/59, $37.3 \%$ ) had biochemical failure, defined as nadir +2 $\mathrm{ng} / \mathrm{mL}$ (16). All patients were biochemically disease free following EBRT.

\section{MR Imaging Technique}

Patients were scanned in a supine position using the body coil for excitation and a pelvic phased array coil (GE Medical Systems, Milwaukee, WI) in combination with a balloon-covered expandable endorectal coil (Medrad, Pittsburgh, PA) for signal reception on a 1.5-Tesla whole body MR scanner (Signa; GE Medical Systems, Milwaukee, WI). The following parameters were used for acquisition of T1-weighted spin-echo MR images of the pelvis: TR/TE 766/8, slice thickness $=5 \mathrm{~mm}$, interslice gap $=1.5 \mathrm{~mm}$, field of view $=24 \mathrm{~cm}$, matrix $256 \times 192$, anteroposterior frequency encoding, and 1 excitation. Thin-section high nominal spatial resolution axial and coronal T2-weighted fast spin-echo images of the prostate and seminal vesicles were acquired with the following parameters: TR/effective TE 5000/96 ms, echo train length $=16$, slice thickness $=3 \mathrm{~mm}$, interslice gap $=0 \mathrm{~mm}$, field of view $=14 \mathrm{~cm}$, matrix $256 \times 192$, anteroposterior frequency encoding (to prevent obscuration of the prostate by endorectal coil motion artifact), and 3 excitations.

\section{Imaging Interpretation}

Two radiologists, with experience in genitourinary radiology, independently reviewed all the images. The radiologists knew patients were treated with external beam radiation therapy for prostate cancer and that all patients had rising PSA values, but had no access to any other clinical or histological information. Images were reviewed at a picture archiving and communication system workstation (Impax; Agfa, Mortsel, Belgium). The following MR imaging data was recorded:

- Presence or absence of post-biopsy hemorrhage on T1-weighted images. Post-biopsy hemorrhage has low signal intensity on T2-weighted images and can be indistinguishable from cancer. On T1-weighted images, however, these foci present high signal intensity and can thereby be differentiated from suspicious areas of low signal intensity on T2-weighted images that represent cancer, therefore improving the specificity of tumor nodule detection.

- Presence or absence of dominant tumoral lesion on T2-weighted images. A study was considered positive if a focal mass-like nodule or crescentic subcapsular focus of low T2 signal intensity was identified within the hemi-prostate (i.e., the left or right side of the gland) (Figures-1 and 2). Because of the known limitations of tumor localization and registration based on sextant biopsy results $(17,18)$, we localized tumor to the hemi-prostate. The limitation of the prostatic sextant as a unit of analysis is illustrated in a prior study of tumor localization with MR imaging and MR spectroscopic imaging, in which the accuracy of imaging for sextant localization was only $67 \%$ (157 of 234 ) to $74 \%$ (173 of 234 ), but that of imaging for tumor lateralization was $75 \%$ ( 80 of 106 ) to $88 \%$ (93 of 106) (19). The difference was, presumably, at least partially due to errors in registration between imaged sections and biopsy specimens. Such errors are likely to be magnified in the irradiated gland because of radiation-induced shrinkage and distortion of tissue.

We opted to describe only the dominant lesion in each patient based on the results of a study by Pucar et al. that demonstrated that clinically significant local recurrence following radiation therapy presents as a single focus at the site of primary tumor (20).

\section{Standard of Reference}

Transrectal ultrasound-guided biopsy was the standard of reference in this study. All but two biopsies were performed at our institution using prostatic nerve blockade. The usual number of specimens that were obtained is 16 , using a systematic approach that targeted the right and left sides of the gland at different levels, as well as suspicious areas seen on ultrasound. We retrospectively reviewed the histopathological reports of all procedures. A report was issued by one of the attending pathologists in our institution for all cases, including the two performed at an outside institution. Samples processed at our institution were fixed in formalin immediately after biopsy and subsequently placed in a block of paraffin wax. Microtome sections were then mounted on a glass slide and stained with hematoxylin and eosin. High molecular weight keratin immunoperoxidase staining was also performed on areas suspicious for adenocarcinoma. 


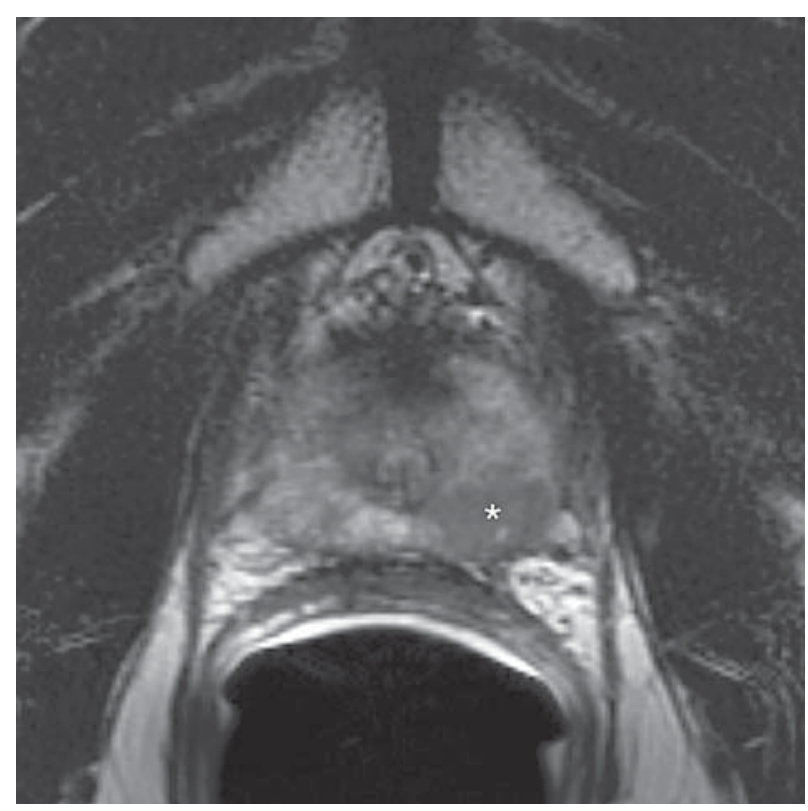

Figure 1 - 61 year-old man with biopsy-proven recurrence of prostate cancer in the left apex and mid-gland 5 years and 6 months after treatment. Axial T2-weighted image (TE/TR 5000/96) shows a focal nodule of low signal intensity in peripheral zone of the left apex of the prostate (asterisk). Both readers interpreted it as a dominant tumoral lesion in left hemi-prostate.

Histopathological evidence of post-treatment effect only was considered a negative result (21).

The presence of cancer on histopathology reports was recorded on a per-sextant basis; however, for the reasons stated above, we determined recurrent cancer to be absent or present in the hemi-prostate.

\section{Statistical Analysis}

When reading $\mathrm{T} 2$-weighted images, our study design called for each reader to only identify the domi-

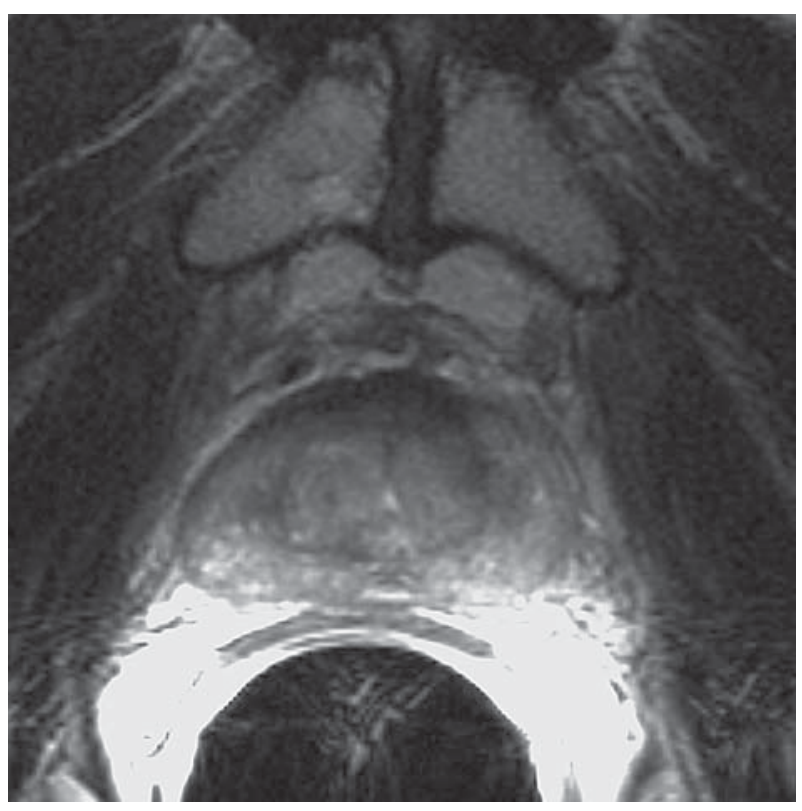

Figure 2 - 63 year-old man with biopsy-proven recurrence of prostate cancer in the right mid-gland and base 4 years and 2 months after treatment. Axial T2-weighted image (TE/TR 5000/96) shows diffuse low signal intensity in the peripheral zone and central gland. Both readers interpreted it as a negative case.

nant side of a lesion whenever it was bilateral (as explained previously within "Imaging Interpretation"). Therefore, there was an inherent a priori constraint to the data format that cancer could not be identified bilaterally. When analyzing whether readers correctly diagnosed cancer, the definition of the dominant side was taken into account according to the design given in Table-1. That is, a positive diagnosis was considered correct if the reader a) correctly diagnosed the patient as having cancer and if so; b) correctly

Table 1 - Definition of outcome categories. A positive diagnosis was considered correct if the reader correctly identified tumor and correctly noted the side of the prostate gland containing cancer. In patients in which tumor was bilateral, the reader was considered correct regardless of which side was indicated as dominant.

\begin{tabular}{|c|c|c|c|c|c|c|c|c|c|c|c|c|c|}
\hline \multirow[b]{2}{*}{ MRI } & \multirow[b]{2}{*}{ Right } & \multicolumn{4}{|c|}{ True Positive } & \multirow{2}{*}{$\begin{array}{c}\text { True Negative } \\
-\end{array}$} & \multicolumn{4}{|c|}{ False Positive } & \multicolumn{3}{|c|}{ False Negative } \\
\hline & & + & + & - & - & & + & + & - & - & - & - & - \\
\hline & Left & - & - & + & + & - & - & - & + & + & - & - & - \\
\hline \multirow[t]{2}{*}{ Biopsy } & Right & + & + & + & - & - & - & - & - & + & + & + & - \\
\hline & Left & + & - & + & + & - & - & + & - & - & + & - & + \\
\hline
\end{tabular}

MRI = magnetic resonance imaging. 
determined the side of the prostate gland containing cancer - if the cancer was bilateral then the reader was considered correct regardless of which side was named dominant. This allowed us to employ simple and robust non-parametric statistical methods while also taking into account whether the correct side of the prostate was diagnosed as containing cancer.

Kappa statistics were used to determine the level of interobserver agreement.

For the purpose of statistical analysis, the patients in this study were divided in two groups, "early" and "late". Patients who had imaging performed within the first 3 years after external beam radiation therapy formed the group called "early". Conversely, the group named "late" included all patients who were imaged three or more years after treatment. This division was based on the results of the studies by Pollack and Vance $(4,5)$, which suggest that identification of cancer in the first two or three years after treatment negatively impacts long-term outcome. Twenty-five patients were imaged within 3 years of treatment and 34 more in the 3 years after therapy.

Because other factors may have inflenced the accuracy of MR imaging, we assessed the similarity in distribution of several variables between these two groups. The Wilcoxon signed rank test was used to assess their distribution with respect to the continuous variables of pre-treatment PSA level, Gleason score, and radiation dose. Gleason score was treated as a continuous variable because of the large number of possible categories and its ordinal quality. Fisher's exact test was used to assess the distribution of patients within the two groups according to the discrete variables D'Amico risk stratification (15), TNM stage, presence or absence of biochemical failure, and the use of neoadjuvant or adjuvant hormonal therapy. The Freeman-Halton extension of Fisher's exact test was used for contingency tables larger than $2 \times 2$.

Logistic regression was used to test for a difference in the accuracy of T2-weighted MR imaging for the detection of cancer in these two groups. The logistic regression model included group ("early" or "late") and diagnosis (presence or absence of cancer on biopsy). The primary test was used for an interaction between group and diagnosis. A significant interaction would indicate a difference in predictive accuracy depending on whether patients were imaged early or late. The model was applied separately to each reader's data.

Table 2 - Patients' characteristics within groups "early" and "late".

\begin{tabular}{lccc}
\hline & Group "Early" & Group "Late" & p Value \\
\hline Pre-treatment PSA* $^{*}$ & $13.9 \mathrm{ng} / \mathrm{mL}(9.43)$ & $21.85 \mathrm{ng} / \mathrm{mL}(26.32)$ & 0.83 \\
T stage & & & 0.69 \\
$1 \mathrm{c}$ & $4 / 22(18 \%)$ & $5 / 32(16 \%)$ & \\
2a-c & $11 / 22(50 \%)$ & $20 / 32(63 \%)$ & \\
3a-b & $7 / 22(32 \%)$ & $7 / 32(22 \%)$ & 0.76 \\
Gleason score* & $6.6(0.58)$ & $6.73(1.04)$ & \\
$\quad$ median (range) & $3+4(3+3$ to $4+3)$ & $3+4(2+3$ to $5+4)$ & \\
D'Amico's risk group & & & \\
high & $10 / 25(40 \%)$ & $16 / 34(47 \%)$ & \\
intermediate & $10 / 25(40 \%)$ & $16 / 34(47 \%)$ & 0.31 \\
low & $5 / 25(20 \%)$ & $2(6 \%)$ & 0.60 \\
Radiation dose* & $75.5 \mathrm{~Gy}(3.27)$ & $15.3 \mathrm{~Gy}(3.73)$ & 0.28 \\
Hormonal therapy & $13 / 25(52 \%)$ & $15 / 34(44 \%)$ & \\
Biochemical failure $^{\Psi}$ & $7 / 25(28 \%)$ & $(44 \%)$ & \\
\hline
\end{tabular}

$*=$ mean (standard deviation), ${ }^{\Psi}=n$ (percentage). 

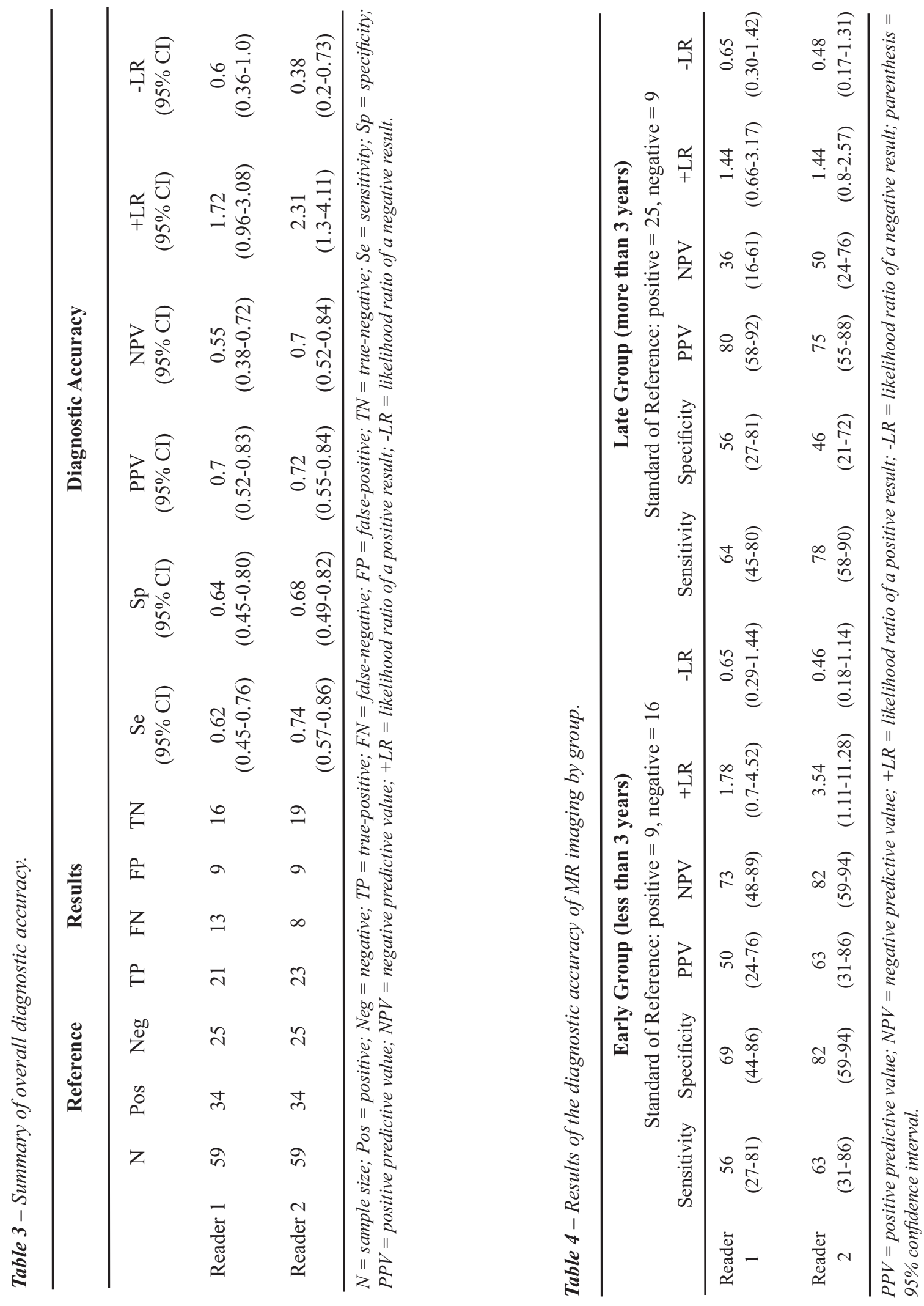
Statistical calculations were performed using SAS/STAT ${ }^{\circledR}$ software v9.1 (SAS Institute Inc., Cary, $\mathrm{NC})$.

\section{RESULTS}

\section{Histopathological Findings}

Forty-one hemi prostates $(41 / 118,34.7 \%)$ in thirty-four patients $(34 / 59,57.6 \%)$ had evidence of cancer on histopathological analysis of transrectal ultrasound-guided biopsy samples. Nineteen patients had recurrence in the right side of the prostate, 8 in the left, and 7 bilaterally. Nine of these patients were part of early post-treatment group $(9 / 25,36 \%)$ and 25 were part of the late post-treatment group (25/34, 73.5\%). All seven patients with tumor detected on both sides of the prostate were part of the latter group.

\section{Patient Characteristics}

There were no statistically significant differences in the balance of patients within groups "early" and "late" according to pre-treatment PSA, clinical stage, Gleason score, D'Amico's risk stratification, radiation dose, neoadjuvant and/or adjuvant hormonal therapy, and evidence of biochemical failure at the time of imaging (Table-2).

\section{MR Imaging Results}

None of the readers detected intra-prostatic hemorrhage on T1-weighted MR images of 13 patients (13/59, $22.0 \%$ ) who underwent biopsy prior to imaging.

Overall, the diagnostic accuracy of T2weighted MR imaging after external beam radiation therapy was $63 \%(37 / 59)$, for reader 1 , and $71 \%$ (42/59), for reader 2 . The sensitivity and specificity of the method was $62 \%(21 / 34)$ and $64 \%(16 / 25)$, for reader 1 , and $74 \%(23 / 31)$ and $68 \%$ (19/28), for reader 2 , respectively. These results, along with the predictive values and likelihood ratios, are detailed in Table-3.

The interobserver agreement was considered good on a per-patient and per-hemi-prostate basis (Kappa coefficient value $=0.59$ and 0.69 , respectively).

The results of the diagnostic accuracy of MR imaging per group, i.e. "early" and "late", are sum- marized in Table-4. For both readers, logistic regression failed to demonstrate a statistically significant difference in the ability of T2-weighted MR imaging to detect cancer based on whether patients were imaged before or after 3 years (reader $1, p=0.86$; reader $2, \mathrm{p}=0.44)$.

\section{DISCUSSION}

Despite our rather liberal criteria for a true positive outcome - identifying tumor within a hemiprostate, even if tumor was bilateral - the results of our study suggest that T2-weighted endorectal MR imaging has low accuracy for the detection of recurrent disease in patients who have undergone definitive treatment with external beam radiation for prostate cancer. The few published studies on MR imaging after EBRT have suggested T2-weighted MR imaging has low to moderate accuracy for the detection of tumor after radiation treatment (9-13). The variability in the numbers reported by the different authors is mostly dependent on three factors: prevalence of disease in the sample, sample size, and statistical analysis methodology.

Pucar et al. enrolled only nine patients, all of which had known recurrence following radiation therapy. Using a sextant approach, they found that MR imaging had a sensitivity of $68 \%$ and specificity of $96 \%$; however, they did not adjust for clustering effects (10). Sala et al. reported areas under the receiver-operating curve (AU-ROC) of $75 \%$ and $61 \%$. They also reported the sensitivity and specificity of MR imaging based on the dichotomization of results measured using a five-point scoring system. These results were very similar to ours (sensitivity $=55-76 \%$, specificity $=65-73 \%)(12)$. In a study that enrolled 22 patients, Rouviere et al. reported a sensitivity ranging from $68 \%$ to $78 \%$ (11). Unfortunately, all but three patients had recurrence, decreasing the significance of the calculation of specificity. Coakley et al. included 21 patients in their study and used the hemi-prostate as unit of analysis. Accounting for clustering effects, they found an AU-ROC of $49 \%$ and $51 \%$ for MR imaging (9). The study by Haider et al. also had a sample size $(n=49)$ and results that were similar to ours, considering the $95 \%$ confidence intervals. Ac- 
cording to their study, MR imaging had a sensitivity and specificity of $58 \%$ and $52 \%$, respectively (13).

The results of all above-mentioned studies, including ours, suggest that MR imaging alone is insufficient for the evaluation of such populations of patients and raises the question of whether other imaging modalities should be used, separately or in conjunction with T2-weighted MR imaging. Among the options available, multiparametric endorectal MR imaging - an approach that incorporates other MR techniques, such as MR spectroscopic imaging, dynamic enhanced MR imaging, and diffusionweighted MR imaging - is promising. Coakley et al. found that a combined approach using MR imaging and MR spectroscopic imaging improved detection of tumor (9). Both Haider and Rouviere reached similar conclusions when they investigated the incremental value of dynamic enhanced MR imaging $(11,13)$. Although these studies support the use of multiparametric MR imaging in patients treated with external beam radiation therapy, the results are preliminary and further investigation with a larger, prospective trial is ultimately required.

As a secondary analysis, we investigated if the accuracy of the MR imaging was influenced by the time interval between radiation treatment and MR imaging. This assumption was based on observation of recovery of the usual zonal anatomy after radiation and/or hormonal therapy and on the results of a study by Pickett et al. (14) that showed recovery of normal metabolism at MR spectroscopic imaging after treatment. We dichotomized the subjects in two groups, those whose MR images were acquired within 3 years after treatment and those whose imaging was performed after 3 years. This decision was supported by the results presented by Pollack and Vance $(4,5)$, which suggest that identification of cancer in the first two or three years after treatment negatively impacts long-term outcome. Our results did not demonstrate an influence of time since treatment on accuracy of MR imaging on a logistic regression model. It is unknown if this in fact represents an accurate picture of the situation or just the result of insufficient power due to a small sample size.

It has been previously demonstrated that hormonal deprivation therapy can significantly reduce tumor volume and decrease peripheral zone signal on T2-weighted images (22), hence having an additional influence in tumor detection on MR imaging. Although it would be interesting to stratify patients in two groups (with and without androgen deprivation therapy) to determine how this would affect our results, it would not possible to obtain any meaningful results of accuracy due to the small number of subjects in each subgroup. This is an issue that must be addressed in future studies.

Our study has limitations. First, it was a retrospective, single institution study. Our results probably are not widely generalizable, as the expertise in MR imaging acquisition and interpretation varies among institutions. Because of our retrospective research design, we probably incurred a sample selection bias, as we included only patients who had a transrectal ultrasound-guided biopsy. It may be expected that the prevalence of recurrent cancer in our population is higher than in the general population of patients treated for prostate cancer with external beam radiation therapy. This could influence our results, as both positive predictive value and negative predictive value are directly related to the prevalence of disease. Although sensitivity and specificity would not be affected. On the other hand, the indications of MR imaging after radiation therapy have not yet been established and more likely the modality will be added to the armamentarium used to investigate patients with suspected local recurrence on the basis of clinical examination or PSA measurements. In fact, our population is representative of this cohort and therefore our results are useful for future standard procedure. Second, our sample size is not large. This has two major effects on our results; it produces a wide $95 \%$ confidence interval for diagnostic accuracy estimations and does not provide us sufficient power to reject the null hypothesis - i.e., the interval of time between treatment and MR imaging does not affect the detection of cancer with T2-weighted MR imaging - if this is fact false (type II error). The wide confidence intervals explain the apparent difference of accuracy between the two readers - not statistically significant - despite relatively good interobserver agreement. Third, transrectal ultrasound-guided biopsy is an imperfect standard of reference. The use of an imperfect standard of reference results in bias of the estimated error rates of MR imaging and the direction of this 
bias is usually downward (23). In our study, which has a relatively large number of patients with disease, i.e. positive biopsy, this bias is probably less significant for the estimation of sensitivity than specificity. It is important, though, to make clear that our results may overestimate the true accuracy of the modality. In this setting, however, overestimation would in fact provide further support to our conclusion: T2-weighted MR imaging appears to have low accuracy for detection of recurrent cancer in patients who underwent external beam radiation therapy. Although whole-mount histopathologic analysis of salvage prostatectomy specimens may be considered a preferable standard of reference, such surgery is infrequently performed in the population we investigated. In addition, this approach also has limitations. In a retrospective study, for instance, it may result in verification bias, as the decision to proceed to surgery is likely influenced by positive results of MR imaging. Our use of the hemi-prostate rather than the prostate sextant as the unit of analysis might also be criticized, although as noted above sextant localization is inaccurate when biopsy is compared to radical prostatectomy specimens, likely due to errors in sextant localization of ultrasound-guided biopsy needles. Such errors are likely to be even greater in the shrunken post-radiation gland. Lateralization should be less subject to such registration problems.

Lastly, the option to consider the reader correct regardless of which side was named dominant in bilateral tumors can also lead to incorrect higher accuracies of the imaging method. We opted for this approach for two reasons: 1) this allowed us to employ simple and robust non-parametric statistical methods while also taking into account whether the correct side of the prostate was diagnosed as containing cancer; and 2) detection of local recurrence in one side, even if disease is bilateral, provides sufficient information for determining management of these patients, as the current standard is to treat them with salvage brachytherapy or salvage prostatectomy (+/- systemic therapy), techniques that treat the entire gland. Irrespective, overestimation of our results supports our conclusion.

In conclusion, T2-weighted MR imaging appears to have low accuracy for detection of recurrent cancer in patients who underwent external beam radiation therapy. Further and larger studies are necessary to confirm these results and to determine if the interval of time between treatment and MR imaging truly has no effect on the accuracy of the method.

\section{ACKNOWLEDGEMENT}

Dr. Antonio C. Westphalen is supported by NIBIB T32 Training Grant 1 T32 EB001631, and RSNA Research \& Education Foundation 2006-07 Research Fellow Grant \#FEL0602 and 2007-2009 Research Scholar Grant \#RSCH0709

\section{CONFLICT OF INTEREST}

None declared.

\section{REFERENCES}

1. Stephenson RA, Stanford JL: Population-based prostate cancer trends in the United States: patterns of change in the era of prostate-specific antigen. World J Urol. 1997; 15: 331-5.

2. Kestin LL, Vicini FA, Ziaja EL, Stromberg JS, Frazier RC, Martinez AA: Defining biochemical cure for prostate carcinoma patients treated with external beam radiation therapy. Cancer. 1999; 86: 1557-66.

3. Moul JW: Prostate specific antigen only progression of prostate cancer. J Urol. 2000; 163: 1632-42.

4. Pollack A, Zagars GK, Antolak JA, Kuban DA, Rosen II: Prostate biopsy status and PSA nadir level as early surrogates for treatment failure: analysis of a prostate cancer randomized radiation dose escalation trial. Int J Radiat Oncol Biol Phys. 2002; 54: 677-85.

5. Vance W, Tucker SL, de Crevoisier R, Kuban DA, Cheung MR: The predictive value of 2-year posttreatment biopsy after prostate cancer radiotherapy for eventual biochemical outcome. Int J Radiat Oncol Biol Phys. 2007; 67: 828-33.

6. Rouvière $\mathrm{O}$ : MR assessment of recurrent prostate cancer after radiation therapy. Radiology. 2007; 242: 635-6; author reply 636-7.

7. Chan TW, Kressel HY: Prostate and seminal vesicles after irradiation: MR appearance. J Magn Reson Imaging. 1991; 1: 503-11.

8. Coakley FV, Hricak H, Wefer AE, Speight JL, 
Kurhanewicz J, Roach M: Brachytherapy for prostate cancer: endorectal MR imaging of local treatmentrelated changes. Radiology. 2001; 219: 817-21.

9. Coakley FV, Teh HS, Qayyum A, Swanson MG, Lu Y, Roach M 3rd, et al.: Endorectal MR imaging and MR spectroscopic imaging for locally recurrent prostate cancer after external beam radiation therapy: preliminary experience. Radiology. 2004; 233: 441-8.

10. Pucar D, Shukla-Dave A, Hricak H, Moskowitz CS, Kuroiwa K, Olgac $\mathrm{S}$, et al.: Prostate cancer: correlation of MR imaging and MR spectroscopy with pathologic findings after radiation therapy-initial experience. Radiology. 2005; 236: 545-53.

11. Rouvière $O$, Valette $O$, Grivolat $S$, Colin-Pangaud $C$, Bouvier R, Chapelon JY, et al.: Recurrent prostate cancer after external beam radiotherapy: value of contrast-enhanced dynamic MRI in localizing intraprostatic tumor--correlation with biopsy findings. Urology. 2004; 63: 922-7.

12. Sala E, Eberhardt SC, Akin O, Moskowitz CS, Onyebuchi CN, Kuroiwa K, et al.: Endorectal MR imaging before salvage prostatectomy: tumor localization and staging. Radiology. 2006; 238: 176-83.

13. Haider MA, Chung P, Sweet J, Toi A, Jhaveri K, Ménard C, et al.: Dynamic contrast-enhanced magnetic resonance imaging for localization of recurrent prostate cancer after external beam radiotherapy. Int J Radiat Oncol Biol Phys. 2008; 70: 425-30.

14. Pickett B, Kurhanewicz J, Coakley F, Shinohara K, Fein B, Roach M 3rd: Use of MRI and spectroscopy in evaluation of external beam radiotherapy for prostate cancer. Int J Radiat Oncol Biol Phys. 2004; 60: 1047-55.

15. D'Amico AV, Whittington R, Malkowicz SB, Schultz D, Blank K, Broderick GA, et al.: Biochemical outcome after radical prostatectomy, external beam radiation therapy, or interstitial radiation therapy for clinically localized prostate cancer. JAMA. 1998; 280: 969-74.

16. Roach M 3rd, Hanks G, Thames H Jr, Schellhammer P, Shipley WU, Sokol GH, et al.: Defining biochemical failure following radiotherapy with or without hormonal therapy in men with clinically localized prostate cancer: recommendations of the RTOG-ASTRO Phoenix Consensus Conference. Int J Radiat Oncol Biol Phys. 2006; 65: 965-74.

17. Wefer AE, Hricak H, Vigneron DB, Coakley FV, Lu Y, Wefer J, et al.: Sextant localization of prostate cancer: comparison of sextant biopsy, magnetic resonance imaging and magnetic resonance spectroscopic imaging with step section histology. J Urol. 2000; 164 : 400-4.
18. Obek C, Louis P, Civantos F, Soloway MS: Comparison of digital rectal examination and biopsy results with the radical prostatectomy specimen. J Urol. 1999; 161: 494-8; discussion 498-9.

19. Scheidler J, Hricak H, Vigneron DB, Yu KK, Sokolov DL, Huang LR, et al.: Prostate cancer: localization with three-dimensional proton MR spectroscopic imaging--clinicopathologic study. Radiology. 1999; 213: 473-80.

20. Pucar D, Hricak H, Shukla-Dave A, Kuroiwa K, Drobnjak M, Eastham J, et al.: Clinically significant prostate cancer local recurrence after radiation therapy occurs at the site of primary tumor: magnetic resonance imaging and step-section pathology evidence. Int J Radiat Oncol Biol Phys. 2007; 69: 62-9.

21. Kestin LL, Goldstein NS, Vicini FA, Mitchell C, Gustafson GS, Stromberg JS, et al.: Pathologic evidence of dose-response and dose-volume relationships for prostate cancer treated with combined external beam radiotherapy and high-dose-rate brachytherapy. Int J Radiat Oncol Biol Phys. 2002; 54: 107-18.

22. Padhani AR, MacVicar AD, Gapinski CJ, Dearnaley DP, Parker GJ, Suckling J, et al.: Effects of androgen deprivation on prostatic morphology and vascular permeability evaluated with $\mathrm{mr}$ imaging. Radiology. 2001; 218: 365-74.

23. Hawkins DM, Garrett JA, Stephenson B: Some issues in resolution of diagnostic tests using an imperfect gold standard. Stat Med. 2001; 20: 1987-2001.

Accepted after revision:

December 12, 2008

\author{
Correspondence address: \\ Dr. Antonio Carlos Westphalen \\ Abdominal Imaging \\ University of California San Francisco \\ 505 Parnassus Avenue, Box 0628, M-372 \\ San Francisco, California, 94143-0628, USA \\ Fax: + 1415 476-0616 \\ E-mail: antonio.westphalen@radiology.ucsf.edu
}




\section{EDITORIAL COMMENT}

The detection of locally recurrent prostate cancer, after external radiation therapy (EBRT), is essential since further treatment options are variable. This includes additional irradiation of the prostate, hormonal therapy, salvage prostatectomy and other new treatment options such as cryosurgery and transrectal high-intensity focused ultrasound. Although several treatment options are available, the management of recurrent prostate cancer after EBRT is a difficult task since all these modalities are associated with high risks of complication (1). For these reasons, precise detection of local recurrence of the tumor is of utmost importance for the management of these patients. The authors performed a retrospective study in order to determine the accuracy of T2-weighted endorectal MR imaging in the detection of prostate cancer after EBRT, and also to investigate the relationship between imaging accuracy and time since therapy. They concluded that "T2-weighted endorectal MR imaging has low accuracy in the detection of prostate cancer after external beam radiation therapy, irrespective of the time since therapy".

As pointed out by the authors in the introduction of their manuscript, tumor depiction with conventional endorectal magnetic resonance imaging in the irradiated gland is of limited value due to treatment-related changes that include prostatic shrinkage, diffuse low T2 signal intensity in the gland, and indistinctness of the normal zonal anatomy $(2,3)$. Since irradiated prostate gland usually appears small and diffusely hypointense on T2-weighted images, magnetic resonance spectroscopic imaging (MRSI), which depicts abnormal metabolism rather than abnormal anatomy, has been shown to be much better technique for the detection of local tumor recurrence and for the demonstration of complete metabolic atrophy (4-6). At our institution, in the last 5 years, we have been using a comprehensive protocol for the detection of recurrent disease in patients treated with EBRT. This protocol consists of a combination of conventional endorectal T2-weighted image with multiparametric functional MRI studies (MRSI, dynamic contrast enhanced and diffusion-weighted images). Using the transrectal guided biopsy as reference, similarly to the authors, we have so far found greater accuracy when using this protocol as compared with conventional T2-weighted images (7).

Regarding the influence of time after EBRT, we found that serial MR spectroscopic imaging is also superior to convention endorectal MRI to demonstrate areas of normal or abnormal metabolism, which can be observed several months after the end of EBRT. Further studies, however, are warranted to confirm this hypothesis.

\section{REFERENCES}

1. Rouvière $\mathrm{O}$ : MR assessment of recurrent prostate cancer after radiation therapy. Radiology. 2007; 242: 635-6; author reply 636-7.

2. Coakley FV, Hricak H, Wefer AE, Speight JL, Kurhanewicz J, Roach M: Brachytherapy for prostate cancer: endorectal MR imaging of local treatmentrelated changes. Radiology. 2001; 219: 817-21.

3. Chan TW, Kressel HY: Prostate and seminal vesicles after irradiation: MR appearance. J Magn Reson Imaging. 1991; 1: 503-11.

4. Scheidler J, Hricak H, Vigneron DB, Yu KK, Sokolov DL, Huang LR, et al.: Prostate cancer: localization with three-dimensional proton MR spectroscopic imaging--clinicopathologic study. Radiology. 1999; 213: 473-80.

5. Yu KK, Scheidler J, Hricak H, Vigneron DB, Zaloudek CJ, Males RG, et al.: Prostate cancer: prediction of extracapsular extension with endorectal MR imaging and three-dimensional proton MR spectroscopic imaging. Radiology. 1999; 213: 481-8.

6. Coakley FV, Teh HS, Qayyum A, Swanson MG, Lu Y, Roach M 3rd, et al.: Endorectal MR imaging and MR spectroscopic imaging for locally recurrent prostate cancer after external beam radiation therapy: preliminary experience. Radiology. 2004; 233: 441-8.

7. van Dorsten FA, van der Graaf M, Engelbrecht MR, van Leenders GJ, Verhofstad A, Rijpkema M, et al.: Combined quantitative dynamic contrast-enhanced MR imaging and (1)H MR spectroscopic imaging of human prostate cancer. J Magn Reson Imaging. 2004; 20: $279-87$.

Dr. Adilson Prando Chief, Department of Radiology Vera Cruz Hospital Campinas, São Paulo, Brazil E-mail: adilson.prando@gmail.com 


\section{EDITORIAL COMMENT}

Detection of post-treatment recurrence of prostate cancer is a challenging situation, both after radical prostatectomy and radiation therapy, since PSA alone may not differentiate between biochemical, local and/or systemic recurrence.

Endorectal MRI (E-MRI), given its intrinsic high contrast resolution, would be the ideal imaging exam for non-invasive detection of local recurrence. However, T2-weighted images of the prostate (the standard imaging technique for prostate MRI) may not suffice for the detection of recurrence, especially after radiation therapy.

The article from Dr. Westphalen et al. reemphasizes the limitations of T2-weighted MRI for the detection of local recurrence after radiation therapy, regardless of the time interval between the procedure and the imaging study.

It should be kept in mind, however, that these results certainly do not diminish the value of E-MRI for the purpose of local recurrence detection. Recent studies have shown that the use of complimentary MRI techniques (namely, spectroscopy and contrast-enhanced dynamic MRI) significantly increases accuracy of the method for the detection of local recurrence, both after radical prostatectomy and after radiation therapy $(1,2)$. Moreover, a recent article correlating MRI and step-section pathology demonstrated that clinically significant local recurrence after radiation therapy occurs at the same site of the primary tumor, so the use of E-MRI before and after treatment could lead to early detection of local recurrence suitable to salvage therapy (3).

Therefore, we can conclude that E-MRI, when used appropriately with the correct dedicated techniques, should be considered in the diagnostic workflow of patients with suspected local recurrence after prostate cancer treatment.

\section{REFERENCES}

1. Pucar D, Sella T, Schöder H: The role of imaging in the detection of prostate cancer local recurrence after radiation therapy and surgery. Curr Opin Urol. 2008; 18: 87-97.

2. Haider MA, Chung P, Sweet J, Toi A, Jhaveri K, Ménard C, et al.: Dynamic contrast-enhanced magnetic resonance imaging for localization of recurrent prostate cancer after external beam radiotherapy. Int J Radiat Oncol Biol Phys. 2008; 70: 425-30.

3. Pucar D, Hricak H, Shukla-Dave A, Kuroiwa K, Drobnjak M, Eastham J, et al.: Clinically significant prostate cancer local recurrence after radiation therapy occurs at the site of primary tumor: magnetic resonance imaging and step-section pathology evidence. Int $\mathrm{J}$ Radiat Oncol Biol Phys. 2007; 69: 62-9.

\section{Dr. Ronaldo Hueb Baroni Institute of Radiology University of São Paulo, USP São Paulo, SP, Brazil E-mail:rbaroni@einstein.br}

